\title{
Texture Granularities
}

\author{
Paul Southam and Richard Harvey \\ University of East Anglia, Norwich, \\ Norfolk, NR4 7TJ, England
}

\begin{abstract}
We introduce three new texture features that are based on the morphological scale-space operator known as the sieve. The new features are tested on two databases. The first, the Outex texture database, contains Brodatz-like textures captured under constant illumination, scale and rotation. The second, the Outex natural scene database, contains images of real-world scenes taken under variable conditions. The new features are compared to univariate granulometries, with which they have some similarities, and to the dual-tree complex wavelet transform, local binary patterns and co-occurrence matrices. The features based upon the sieve are shown to have the best overall performance.
\end{abstract}

\section{Introduction}

Granulometries [10] have had a long history in texture analysis. They have been used for the analysis of digital mammograms 2], radiographic imaging of lungs [17] and Diatom classification [16. Univariate granulometries comprise varyingscale morphological openings and closings applied in parallel using a fixed shape structuring element, scaled by a parameter. It is known [1], that the shape of the structuring element affects texture classification. This poses in interesting question, is it the shape of the structuring element that is important or the analysis over scale? Until recently it was not possible to separate these two criteria because the shape of the filter is fixed. However, by using a different class of mathematical morphology filters called sieves, it is possible to analyse an image by scale without the filter imposing a shape - a technique we explore in this paper.

First we justify our evaluation methods and choose from the many texture databases available. Next we introduce sieves and show how they can provide new texture filters. In the final sections we evaluate the performance of these systems and compare them to a number of benchmark systems including granulometries.

\section{Databases}

This paper uses Outex [1] which has recently become regarded as the best available framework for evaluating texture [15. It contains several tasks. Here we use TC_00000 which is the texture classification task using textures imaged under consistent conditions (perpendicular to the surface, fixed scale, rotation and illumination and so on). Examples of these textures are shown in Figure2. Stylised 


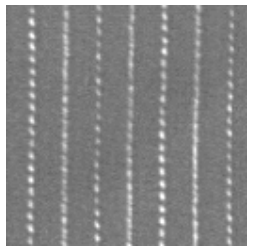

Class A

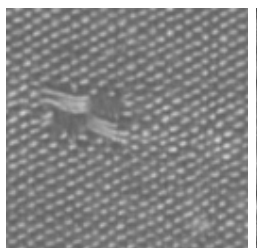

Class B

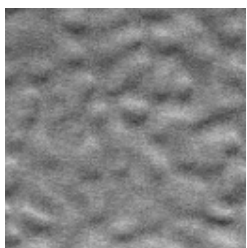

Class C

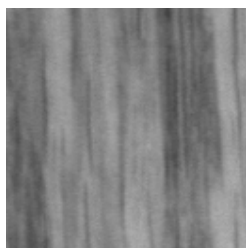

Class D

Fig. 1. Various Outex samples used in Outex task TC_00000
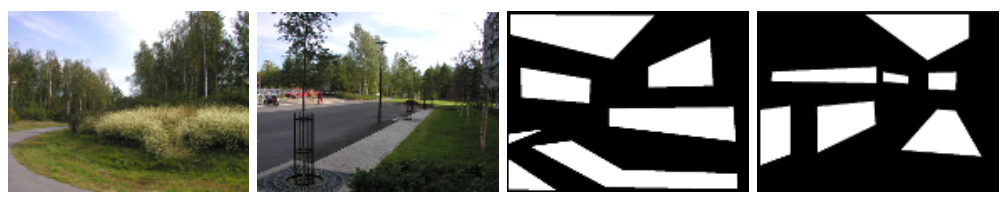

Fig. 2. Various Outex natural scene images (left) and hand-segmented ground-truth (right)

textures such as these are extremely common in the literature (Brodatz [6] and MeasTex [14] are examples) and automatic texture classifiers are known to perform extremely well on such data ( 91$]$ for example). Unfortunately, so far, the performance of such systems has not extrapolated to real-world scenes ([8] and [13]). The natural scenes Outex database contains 20 colour images $(2272 \times 1704$ pixels) of real-world scenes taken with a digital camera under varying illumination and orientation. The view is said to be "roughly consistent, simulating a navigating vehicle" [13. There five defined texture classes, sky, trees, grass, road and buildings which are defined through hand-labelled regions in ground-truth images. Examples of the scene and ground truth images are shown in Figure 2 .

\section{Sieve}

Sieves are described as a one-dimensional non-linear scale-space decomposition algorithm in [5] and are extended to $n$-dimensions in [3] by adopting techniques from graph morphology. Sieves use morphological scale-space operators, specifically openings and closings, or combinations of them, to filter an input signal by removing extrema of specific scale. They apply flat structuring elements to an input signal which, unlike conventional morphological operators such as those used in granulometries, have a fixed size but varying shape. A stated advantage of this approach is that the shape of the structuring element is not visible in filtered signal.

The sieve performs a decomposition by scale via the structure shown in Figure 3. At each stage the filtering operator $\varphi$ removes extrema of only that scale. 


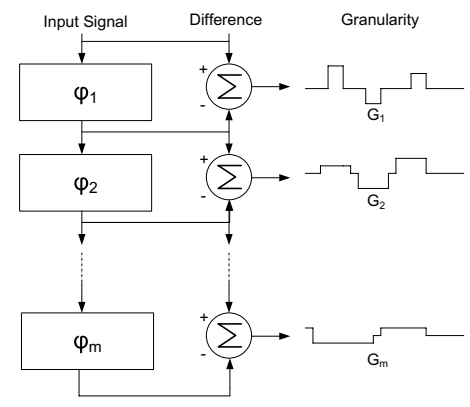

Fig. 3. The structure of a $1 \mathrm{D}$ sieve decomposition where $\varphi$ is a filtering operator chosen from a set 5]3. Non-zero regions in the output are called granules and the set of granules is called the granularity domain in an analogy to granulometries.

At the first stage $\varphi_{1}$ removes extrema of scale 1 (removed extema are called granules $), \varphi_{2}$ removes extrema of scale 2 and so on until the maximum scale $m$ (which is the number of pixels in the image). This serial structure can be contrasted with the parallel structure used in granulometries. Because objects in images are often delineated by iso-intensity contours, sieves have been applied to image segmentation tasks in which semantically meaningful objects are removed at a specific (typically higher) scale. At lower scales the sieve can been seen to remove at first image noise then textural information. Figure 4 shows a example sieve decomposition of an image using a 2D $M$-filter sieve.
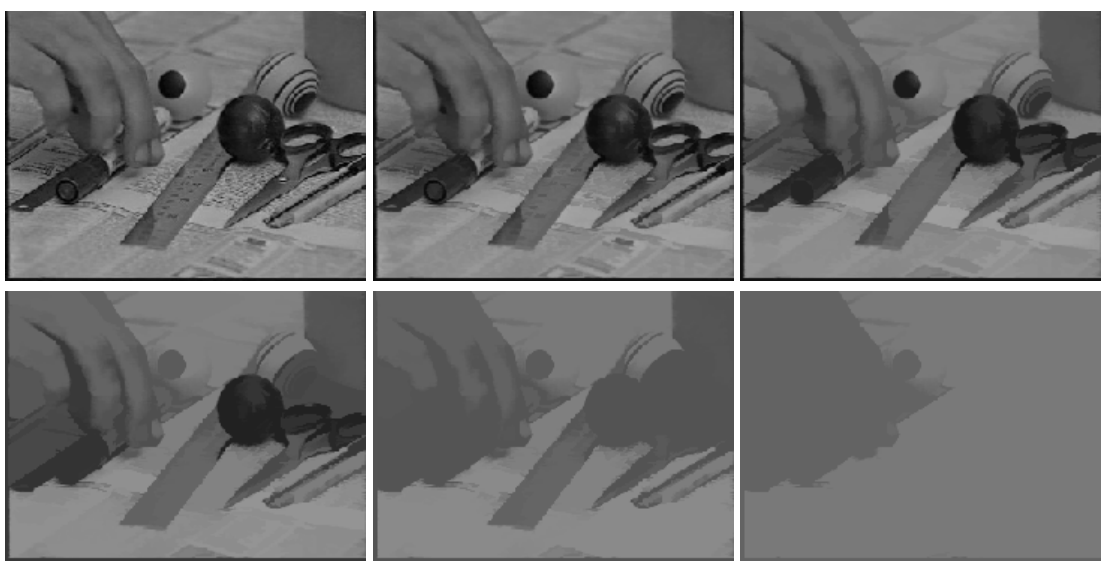

Fig. 4. An original image (top left) sieved to scales, reading left-to-right, top-to-bottom, 15, 251, 2500, 8500 and 25000 . Each image has fewer intensity extrema than its predecessor. A full decomposition may be summed to re-create the original thus the sieve is a transform of the original image. 


\section{Methods}

Here we introduce three new sieve-based texture features. All the new methods are based on granules. In [3], granule images are defined as the difference between successive sieve outputs, $G_{n}=\mathcal{S}_{n}-\mathcal{S}_{n-1}$ where $\mathcal{S}_{n}$ is the $n$th stage in the serial structure shown in Figure 3 . There are thus a great number of granule images. The $G_{n}$ are a transform of the texture which can be reconstructed through a simple summation. Here, each texture image is sieved to a few scales, $\left[s_{1} \ldots s_{N}\right]$ where $\log _{10} s_{n}$ are equispaced between 0 and $\log _{10} P$, where $N=5$ and $P=30$ are chosen to remove all textural information from all images. The difference between these images are termed channels, $C_{n}=\mathcal{S}_{s_{n}}-\mathcal{S}_{s_{n-1}}$. The intensity of the granule, or channel, images as a function of scale is an indicator of the scaledistribution of the texture features. The mean, standard deviation and skewness of the magnitude of the granule images make suitable features.

In the first method $\varphi$ is a 2D $M$-filter [3] which filters the image using an morphological opening followed by a morphological closing in one operation. This produces bi-polar granule images that are invariant to simple rotation of the texture image.

In the second method $\varphi$ is a $1 \mathrm{D}$ recursive median filter [4] where each image is sieved at orientations of $\pm 30^{\circ}, \pm 60^{\circ}, \pm 90^{\circ}$ which produces bi-polar granule images which are sensitive to the image rotation.

The final method (oc-sieve) is similar to granulometries because it uses two sieves: one using an opening filter (o-sieve) and the other using a closing filter $(c$ sieve). This produces twice the number of granule images as in the first method, one set of positive granule images derived from only the image maxima and another set derived from only the image minima. The results from this method highlights the difference between processing maxima and minima in on one bipolar operation as in the 2D M-sieve, and separately using the uni-polar $o$-sieve and $c$-sieve.

For comparison we also implement features based on, the dual-tree complex wavelet transform [7, Local binary pattern analysis [12, co-occurrence matrices, and three granulometic methods with different sized structuring elements. The first granulometic method uses a combination of disc and vertical structuring elements (denoted GDV subsequently) as these are known to perform best on a selection of Brodatz textures [1]. We also implement a rotation-invariant granulometic method based upon disc and square structuring elements (denoted GDS) for comparison with the 2D sieve; and a rotation-sensitive method using line structuring elements at angles of $\pm 30^{\circ}, \pm 60^{\circ}, \pm 90^{\circ}$ (denoted GLA) for comparison with the $1 \mathrm{D}$ sieve. In all the granulometic methods the size of the structuring element is within the scales used for a sieve channel. Features are formed from the means, standard deviations and skewnesses of the differences between successive openings and closings of the image as with the $o c$-sieve method.

In all the methods the results are always improved by re-projecting the feature vectors via principal component analysis (PCA) with the further possibility to reduce feature dimension. During classification the Euclidean distances are measured between each feature vector in the PCA space and a $k$ nearest neigh- 
bour classifier is used to predict the test class. For test set TC_00000 it is known that $k=1$ is optimal [15] (indicating, incidentally, that TC_00000 could be improved with more data). For the natural scenes data we find $k=3$ to be the best.

\section{Results}

Outex_TC_00000 comprises 480 images (24 texture classes each with 20 images). There are 100 standard learning tasks. Each has 240 training and 240 test images. Table 1 shows the mean success rate over the 100 tasks using the nearest neighbour classifier with a Euclidean distance measure on the feature vector after PCA (the final column of Table1 (left) is the size of the PCA vector without truncation). Note that the success rate of the LBP method differs from [12] because, for consistency, we are not using their histogram distance measure.

These results show (as in [1]) that oriented structuring elements (GLA and GDV) are the best among the granulometric methods. However both the DTCWT and 1D sieve score better in this test and are the best performing overall. The DTCWT produces eight sub-bands per level so three levels produce $8+8^{2}+8^{3}$ bands hence 336 features (mean and standard deviation of the absolute value). The 1D-sieve feature (and the GLA feature) is formed over only five channels (at scales 0 to 1,1 to 2,2 to 5,5 to 13 and 13 to 30) and six orientations which is only 30 components so we can afford to compute the mean, standard deviation and skewness which still retaining fewer features than the DTCWT. The 2D- and oc-sieves have channels of the same scales as the GLA and 1D-sieve. The 2D-sieve hence as 15 features (mean, standard deviation and skewness per channel) and the oc-sieve has double the number because it has channels at positive and negative scales. LBP has 256 features as described in [12]. The cooccurrence matrices have 12 features representing energy, inertia, entropy and homogeneity at orientations of $0^{\circ}, 90^{\circ}$ and $45^{\circ}$. GDV has 48 features which are the mean, standard deviation and skewness over the five standard channels for the vertical element. The circular element structuring elements must have integer radii which leads us to choose only three scales for the discs (radii 1,2 and $3)$. GDS uses the same discs and squares with sides of 1,2,4 and 5 pixels.

Table 1 (right) shows the result of running McNemar's test at a significance of $\alpha=0.05$. The entries show the number of times, out of a 100 runs, that the test allows us to reject the null hypothesis. Ignoring any arguments about Bonferroni adjustments, this is a crude measure of whether the texture classifiers differ significantly. The 1D-sieve, DTCWT, GDV and GLA have very similar performance with 1D-sieve and DTCWT the best performing. Using PCA to reduce feature dimensionality improves the $1 \mathrm{D}$ sieve success rate to 0.999 which is the same as the DTCWT but using only 40 features. The effect of applying PCA to the DTCWT incurs no performance increase but maintains a 0.999 success rate using 77 features.

For the Outex natural scene database, features are generated from each handsegmented region in the 20 images in this database. Not all texture methods are 
Table 1. Left: $\bar{x}=1-e$ (success rate), max and min success rate out of the 100 trials, standard deviation $\sigma$, (for standard error divide by ten) and number of features $f$ for Outex test suite Outex_TC_00000. Right: The number of times out of the Outex_TC_00000 100 trials that, under McNemar's test, we can confidently ( $\alpha=0.05)$ reject the null hypothesis that the two data distributions are drawn from the same source.

\begin{tabular}{|c|c|c|c|c|c|}
\hline & $\bar{x}$ & 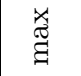 & 龵 & $\sigma$ & $\mathrm{f}$ \\
\hline 1D sieve & 0.998 & $\overline{1}$ & 0.988 & 0.0034 & 90 \\
\hline GLA & 0.995 & 1 & 0.975 & 0.0054 & 90 \\
\hline 2D sieve & 0.954 & 0.975 & 0.929 & 0.0106 & 15 \\
\hline GDS & 0.986 & 1 & 0.954 & 0.0081 & 42 \\
\hline GDV & 0.995 & 1 & 0.975 & 0.0046 & 48 \\
\hline oc-sieve & 0.970 & 0.988 & 0.938 & 0.0105 & 30 \\
\hline DTCWT & 0.999 & 1 & 0.988 & 0.0019 & 336 \\
\hline LBP & 0.986 & 0.996 & 0.967 & 0.0007 & 256 \\
\hline cO-Occ & 0.946 & 0.983 & 0.900 & 0.0015 & 12 \\
\hline
\end{tabular}

\begin{tabular}{|c|c|c|c|c|c|c|c|c|c|}
\hline & 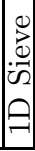 & 顿 & $\begin{array}{l}0 \\
\stackrel{0}{w} \\
\stackrel{0}{\omega} \\
\stackrel{2}{N}\end{array}$ & 官 & 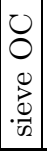 & $\begin{array}{l}5 \\
5 \\
0 \\
0 \\
0 \\
0\end{array}$ & $\frac{0}{9}$ & $\begin{array}{l}0 \\
0 \\
0 \\
1 \\
0 \\
0\end{array}$ & 它 \\
\hline 1D sieve & 0 & 0 & 95 & 8 & 61 & 0 & 10 & 96 & 0 \\
\hline GLA & - & 0 & 88 & 3 & 39 & 0 & 3 & 94 & 0 \\
\hline 2D sieve & - & - & 0 & 54 & 14 & 98 & 43 & 2 & 87 \\
\hline GDS & - & - & - & 0 & 16 & 11 & 1 & 71 & 2 \\
\hline sieve OC & - & - & - & - & 0 & 64 & 8 & 25 & 82 \\
\hline DTCWT & - & - & - & - & - & 0 & 12 & 96 & 0 \\
\hline LBP & - & - & - & - & - & - & 0 & 67 & 3 \\
\hline Co-occ & - & - & - & - & - & - & - & 0 & 94 \\
\hline GDV & - & - & - & - & - & - & - & - & 0 \\
\hline
\end{tabular}

easily applicable to this set because the regions are hand-drawn so, for example, wavelet support-regions are not guaranteed to fit the ground-truth. We therefore restrict the comparison to sieves and granulometries because, for these, we can generate the filtered images, apply the hand-segmented region as a mask, and generate a feature for each region. There are a total of 91 labelled regions which is too few for holdout. Therefore we use leave-out-one cross-validation. Table 2 (left) shows the success rate across all classes, with a knn classifier $(k=3$, Euclidean distance). Also shown is the number of samples per class. GLA and GDV perform quite well as expected. However it appears that both are quite fragile - certain types of texture are difficult to classify with these methods. The best overall performers are the oc- and 2D-sieves.

Table 2 (right) shows the $p$-values obtained under McNemars test where $p>3.84$ allows the null hypothesis (that the two classifiers are indistinguishable) to be rejected at $\alpha=0.05$ with oc-sieve probably the best performing overall.

\section{Conclusions and Discussion}

The Outex_TS_00000 test suite has images of texture at a large scale captured under consistent illumination and rotation. This test set is highly representative of the data used to evaluate the majority of texture classifiers over the past thirty years. Scholarly interest in granulometries seems to have declined recently but here we show that granulometries are among the best-performing particularly when using directional structuring elements. The overall trend in these results is 
Table 2. Left: Number of samples per class, mean success rate for each class and overall mean success rate for the Outex natrual scene database. Right: McNemar's p-values $(p>3.84$ is the threshold for rejecting the null hypothesis at $\alpha=0.05)$

\begin{tabular}{|c|c|c|c|c|c|c|c|}
\hline & 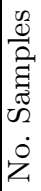 & 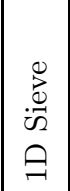 & $\overleftrightarrow{\leftrightarrows}$ & $\begin{array}{l}0 \\
\stackrel{0}{0} \\
\cdot \vec{N} \\
\stackrel{\sim}{N}\end{array}$ & $\tilde{\tilde{U}}$ & $\overrightarrow{0}$ & $\begin{array}{l}0 \\
0 \\
0 \\
0 \\
\frac{0}{2}\end{array}$ \\
\hline sky & 14 & 0.79 & $\overline{1}$ & 1 & 0.93 & $\overline{1}$ & 1 \\
\hline tree & 17 & 0.59 & 0.76 & 0.82 & 0.76 & 0.71 & 0.88 \\
\hline bush & 15 & 0.20 & 0.27 & 0.53 & 0.60 & 0.33 & 0.53 \\
\hline grass & 20 & 0.60 & 0.70 & 0.65 & 0.70 & 0.75 & 0.80 \\
\hline road & 16 & 0.38 & 0.56 & 0.88 & 0.56 & 0.63 & 0.69 \\
\hline building & 9 & 0 & 0.22 & 0.33 & 0.11 & 0.33 & 0.56 \\
\hline mean & & 0.42 & 0.59 & 0.70 & 0.60 & 0.62 & 0.7 \\
\hline
\end{tabular}

\begin{tabular}{|c|c|c|c|c|c|c|}
\hline & 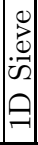 & $\underset{త}{\overleftrightarrow{G}}$ & 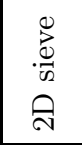 & 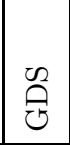 & $\begin{array}{l}0 \\
0 \\
0 \\
\stackrel{u}{\omega} \\
.\end{array}$ & $\vec{\theta}$ \\
\hline 1D sieve & 0 & 5.63 & 14.69 & 7.03 & 19.31 & 8.65 \\
\hline GLA & - & 0 & 3.12 & 0.05 & 5.33 & 0.10 \\
\hline 2D sieve & - & - & 0 & 2.04 & 0.31 & 1.75 \\
\hline GDS & - & - & - & 0 & 3.70 & 0.06 \\
\hline sieve OC & - & - & - & - & 0 & 3.70 \\
\hline GDV & - & - & - & - & - & 0 \\
\hline
\end{tabular}

that rotationally invariant methods such as GDS, the oc-sieve and the 2D-sieve perform poorly, implying that, when the orientation of the texture is known, then there is no advantage to rotationally invariant features 15. The DTCWT is also directionally sensitive and performs well but it uses a large number of features compared to the 1D-sieve.

When the rotation of the texture is unknown, a more realistic situation for unconstrained computer vision, then directional texture features are likely to perform poorly. For this reason one seeks systems that are able to operate on natural scenes. In the Outex natural scene database the 2D-sieves and oc-sieves have the highest success rates (and are usually the best performing on individual classes) followed by the GDV and GDS. The 1D-sieve, which was one of the best performing methods on Outex_TS_00000, is now the worst which is a concern for those who wish to extrapolate from the performance on stylised texture images to reality.

\section{References}

1. G. Ayala and J. Domingo. Spatial size distributions: applications to shape and texture anaylsis. IEEE Transactions on Pattern Analysis and Machine Intelligence, 23(12):1430-1442, 2001.

2. S. Baeg, S. Batman, E. Dougherty, V. Kamat, N. Kehtarnavaz, S. Kim, A. Popov, K. Sivakumar, and R. Shah. Unsupervised morphological granulometric texture segmentation of digital mammograms. Electronic Imaging, 8(1):65-75, 1999.

3. J. Bangham, R. Harvey, P. Ling, and R. Aldridge. Morphological scale-space preserving transforms in many dimensions. Journal of Electronic Imaging, 5:283-299, 1996.

4. J. Bangham, P. Ling, and R. Young. Multiscale recursive medians, scale-space, and transforms with applications to image-processing. IEEE Trans Image Processing, 5(6):1043-1048, June 1996. 
5. J. A. Bangham, P. Chardaire, C. J. Pye, and P. D. Ling. Multiscale nonlinear decomposition: The sieve decomposition theorem. IEEE Transactions on Pattern Analysis and Machine Intelligence, 18(5):529-539, May 1996.

6. P. Brodatz. Textures : A Photographic Album. New York : Dover, 1966.

7. S. Hatipoglu, K. Mitra, and N. Kingsbury. Texture classification using dual-tree complex wavelet transform. In Image Processing and its Applications, pages 344 347. IEE, 1999.

8. P. Howarth and S. Ruger. Evaluation of texture features for content-based image retrieval. In International Conference on Image and Video Retrieval (CIVR) 2004, pages 326-324, 2004.

9. B. Manjunath and W. Ma. Texture features for browsing and retrieval of image data. IEEE Transactions on Pattern Analysis and Machine Intelligence, 18(8):837842, 1996.

10. G. Matheron. Random sets and integral geometry. John Wiley, New York, 1975.

11. T. Ojala, T. Maenpaa, M. Pietikainen, J. Viertola, J. Kyllonen, and S. Huovinen. Outex - new framework for empirical evaluation of texture analysis algorithms. In Proc. 16th International Conference on Pattern Recognition, Quebec, Canada, volume 1, pages 701-706, 2002.

12. T. Ojala, M. Pietikanien, and D. Harwood. A comparative study of texture measures with classification based on feature distributions. Pattern Recognition, 29:5159, 1996.

13. M. Pietikainen, T. Nurmela, T. Maenpaa, and M. Turtinen. View-based recognition of real-world textures. Pattern Recognition, 37:313-323, 2004.

14. G. Smith and I. Burns. Measuring texture classification algorithms. Pattern Recognition Letters, 18:1495-1501, 1997.

15. P. Southam and R. Harvey. Compact rotation-invariant texture classification. In International Conference on Image Processing (ICIP 2004), pages 3033-3036. IEEE, 24-27 Oct 2004.

16. E. Urbach, J. Roerdink, and M. Wilkinson. Connected rotation-invariant sizeshape granulometries. In 17th International Confernece on Pattern Recognition (ICPR) 2004,, volume 1, pages 688-691, 2004.

17. M. Vanrell and J. Vitria. Mathematical morphology, granulometries, and texture perception. Image algebra and morphological image processing IV, SPIE-2030:152161, 1993. 